\title{
О ПРЕДЕЛЬНОЙ ЧУВСТВИТЕЛЬНОСТИ ФОТОСЕНСИБИЛИЗИРУЕМЫХ ГАЗОВЫХ СЕНСОРОВ НА ОСНОВЕ ПОВЕРХНОСТНОГО ПЛАЗМОННОГО РЕЗОНАНСА НА ПЛЕНКАХ ЙОДИДА СЕРЕБРА
}

\author{
А.В. Крайский ${ }^{1}$, А.А. Крайский${ }^{2}$ В.В. Савранский ${ }^{3}$, М.А. Кононов ${ }^{3}$ \\ ${ }^{1}$ Физический институт им.П.Н. Лебедева РАН, Ленинский просп. д.53, Москва, Российская Федерация, \\ 119991 \\ E-mail: kraiski@sci.lebedev.ru \\ ${ }^{2}$ Независимый исследователь, ул. Старобитцевская д.11, кв. 156, Москва, Российская Федерация, 117628 \\ E-mail: vasi113579@gmail.com \\ ${ }^{3}$ Институт общей физики им. А.М. Прохорова РАН, ул. Вавилова, д. 38, Москва, Российская Федерация, \\ 119991 \\ E-mail: savran@nsc.gpi.ru
}

В работе на основе молекулярно-кинетической теории проведен анализ опубликовнной ранее работы (С.В. Виноградов, М.А. Кононов, В.В. Савранский, и др. Квантовая электроника, 2003. T. 33. №8. С. 711-713) о влиянии поверхностной оптической сенсибилизации на величину резонансного угла поверхностного плазмонного резонанса сенсора. В ней было показано, что следовые концентрации молекул сенсибилизатора Арсеназо 3 в воздухе при освещении лазером с длиной волны 543,7 нм исследуемой с помощью поверхностного плазмонного резонанса трехслойной поверхности, верхний слой которой состоит из нанокристаллов йодидов серебра, вызывают на поверхности этих нанокристаллов выделение кластеров металлического серебра, занимающие около $15 \%$ площади нанокристалла, содержсацие по оценкам настоящей работы около 104 атомов серебра при адсорбции на этом нанокристалле одной молекулы красителя, что приводит к изменению резонансного угла. На основе проведенного анализа объяснен линейный по времени проведения эксперимента на начальном этапе рост отклонения резонансного угла и оценено, что вблизи поверхности сенсора концентрация, вызывающая такое почернение 10\% общего количества нанокристаллов, составляет от $4 \cdot 10^{5} \mathrm{~cm}^{-3}$ за 1 с до $4 \cdot 10^{3} \mathrm{~cm}^{-3}$ за $100 \mathrm{c}$, что относится к лучиим опубликованным достижениям, и конкурирует с обонянием живых организмов. Приводится краткий обзор методов и результатов, полученных для следовых концентраций веществ порядка единиц ррt.

Ключевые слова: оптическая сенсибилизация, молекулярно-кинетическая теория, иодид серебра, диффузия, тяжелая молекула, следовые количества, воздух

\section{ON THE LIMITING SENSITIVITY OF THE BEING PHOTOSENSITIZED SENSORS OF GAS BASED ON SURFACE PLASMON RESONANCE ON SILVER IODIDE FILMS}

\author{
A.V. Kraiski ${ }^{1}$, A.A. Kraiski ${ }^{2}$, V.V. Savransky ${ }^{3}$, M.A. Kononov ${ }^{3}$ \\ ${ }^{1}$ P.N. Lebedev Physical Institute of RAS, Leninsky prosp. 53, Moscow, Russian Federation, 119991 \\ E-mail: kraiski@sci.lebedev.ru \\ ${ }^{2}$ Starobitsevskaya str.11-156, Moscow, Russian Federation, 117628 \\ E-mail: vasil13579@gmail.com \\ ${ }^{3}$ Prokhorov General Physics Institute of RAS, Vavilova str. 38, Moscow, Russian Federation, 119991 \\ E-mail: savran@nsc.gpi.ru
}


In the article, on the basis of molecular kinetic theory, an analysis of the previously published article (S.V. Vinogradov, M.A. Kononov, V.V. Savransky, S.I. Valyansky, M.F. Urbaitis. Quantum Electronics. 2003. V. 33. N. 8. P. 711-713) on the effect of surface optical sensitization on the value of the resonance angle of the surface plasmon resonance of the sensor. The sensor represented a three-layer film structure of silver - aluminum oxide - silver iodide (top layer). The top layer consists of silver iodide nanoclusters. In the study of this film structure using surface plasmon resonance, it was shown that in the presence of a trace concentration of molecules of the Arsenazo 3 sensitizer in the air and when illuminated by a laser with a wavelength of $543.7 \mathrm{~nm}$, the resonance angle changes. This is a consequence of the liberation of metallic silver clusters on silver iodide nanocrystals, which occupy about $15 \%$ of the nanocrystal area, containing, according to the estimates of this work, about 104 silver atoms upon adsorption of one dye molecule on this nanocrystal.Based on the analysis performed, the linear in time of the experiment was explained at the initial stage of the increase in the deviation of the resonance angle and it was estimated that near the sensor surface the concentration causing such blackening of $10 \%$ of the total number of nanocrystals is from $4 \cdot 10^{5} \mathrm{~cm}^{-3}$ per $1 \mathrm{~s}$ to $4 \cdot 10^{3} \mathrm{~cm}^{-3}$ for $100 \mathrm{~s}$, which is among the best published achievements, and competes with the sense of smell of living organisms. A brief overview of the methods and results obtained for trace concentrations of substances of the order of ppt units is given.

Key words: optical sensitization, molecular kinetic theory, silver iodide, diffusion, heavy molecule, trace amounts, air

Для цитирования:

Крайский А.В., Крайский А.А., Савранский В.В., Кононов М.А. О предельной чувствительности фотосенсибилизируемых газовых сенсоров на основе поверхностного плазмонного резонанса на пленках йодида серебра. Рос. хим. ж. (Ж. Рос. хим. об-ва). 2021. T. LXV. № 4. C. 42-48

For citation:

Kraiski A.V., Kraiski A.A., Savransky V.V., Kononov M.A. On the limiting sensitivity of the being photosensitized sensors of gas based on surface plasmon resonance on silver iodide films. Ros. Khim. Zh. 2021. V. 65. N 4. P. 42-48

\section{ВВЕДЕНИЕ}

Определение следовых (т.е. предельно малых) концентраций летучих веществ в различных применениях не теряет актуальности многие годы. Определение таких веществ с помощью обоняния весьма специфично. Чувствительность человека к тринитробутилтолуолу составляет до $\sim 10^{7}$ мол/ $\mathrm{cm}^{3}$, собаки к молекулам масляной кислоты - $10^{4}$ мол/ $\mathrm{cm}^{3}$, а самца бабочки тутового шелкопряда к феромонам самки - 100 мол/см ${ }^{3}$ [1]. К уксусной кислоте, чувствительность человека составляет $\sim 5 \cdot 10^{13}$ мол/ $\mathrm{cm}^{3}$, а собаки - 5·10 менных способов определения следовых количеств летучих веществ находится на уровне $10^{9}$ мол/см ${ }^{3}$ [2].

О публикациях. Поисковая база Google academic на июль 2021 г. дает за последние 15 лет около 18700 ссылок, а для концентраций на уровне ppt только обзорных работ имеется 81. Такие концентрации реальны при выявлении взрывчатых веществ [3]. Параметры насыщенных паров в этой работе: для нитроглицерина $-10^{6} \mathrm{ppt}$, для тротила - $10^{4}$ ppt, для гексогена - 1 ppt. В [4] рассматриваются различные методы анализа. Отмечается, что рентгеноспектральный эмиссионный анализ позволяет определять содержание нескольких нг, а методы изотопного разбавления - $10^{-9}-10^{-17}$ г.
В обзоре [5] приведены минимальные концентрации для $\mathrm{Pb}$ и $\mathrm{Cd}$, полученные при помощи лазерно-искровой эмиссионной спектрометрии. К достоинствам ее относится малость проб (размер порядка 6-10 мкм, масса материала $10^{-9}$ г) и мобильность приборов. В [6] рассмотрены проблемы определения следовых количеств ароматических углеводородов в средах при концентрациях порядка 100 ppt. При контроле воздуха важно содержание $\mathrm{NO}_{2}$ с пороговым значением около 50 ppt [7]. $\mathrm{O}$ возможности определения $\mathrm{HCl}$ и некоторых азотосодержащих неорганических кислот до уровня 16-50 pptv сообщается в [8]. В [9-14] исследовались сенсоры на основе изменения электрических свойств. В [9] для сенсора на переходе AlGaN/GaN для $\mathrm{NO}$ и $\mathrm{NO}_{2}$ рабочий диапазон концентраций составляет 10 - 800 ppm, а для $\mathrm{NH}_{3} 150$ ppb -15 ppm. $\mathrm{B}$ [10] продемонстрирован отклик изменения проводимости графена на присутствие в потоке чистого $\mathrm{N}_{2}$ примеси $\mathrm{NO}$ с концентрацией 0,158 ppt (концентрация молекул порядка $4 \cdot 10^{6} \mathrm{~cm}^{-3}$ ). В обзоре [11] показано, что электропроводность сенсора на основе углеродных одностенных нанотрубок в присутствии газа, содержащего $\mathrm{NO}_{2}$ с концентрацией 2ppm, селективно изменяется. В [12] на переходе нанопроволоки $\mathrm{SnO}_{2}$ - монослой графена 
показан предел регистрации $\mathrm{NO}_{2}$ до 24 ppt. Ha n-p$\mathrm{n}$ гетеропереходе углеродные нанотрубки - нанопроволоки $\mathrm{SnO}_{2}$ предел регистрации $\mathrm{NO}_{2}$ достиг $20 \mathrm{ppt}$ [13]. В [14] на переходе углеродные многостенные нанотрубки - нанопроволоки $\mathrm{SnO}_{2}$ получен предел обнаружения $\mathrm{NO}_{2}$ до 0,68 ppt.

Широкое распространение получил специфический метод, называемый по-русски «гигантское комбинационное рассеяние света» (ГКРС или ГКР), а по-английски - surface-enhanced Raman scattering (SERS). Интенсивность линий рассеяния в нем до 10-11 порядков больше, чем в обычном. Это зависит от подложки, содержащей тестовые молекулы. В [15] обсуждаются свойства наиболее распространенных металлических $(\mathrm{Ag}, \mathrm{Cu}$ и $\mathrm{Au})$ подложек и коммерчески доступных подложек. SERS нашел применения в аналитике в различных областях. В [16] была оценена возможность применения наночастиц Au и Ag для обнаружения взрывчатых веществ методом SERS. На наночастицах $\mathrm{Au}$, нанесенных на листы $\mathrm{Al}$, было обнаружено $7,8 \cdot 10^{-18}$ г тротила. В [17] авторы продемонстрировали сверхчувствительное обнаружение биологических видов и загрязнителей окружающей среды, показав, что основания ДНК тимина и аденина, могут быть обнаружены в воде в субфемтомолярных концентрациях без дополнительных процессов мечения.

Ранее [18] было показано влияние поверхностной оптической сенсибилизации (ПОС) на поверхностный плазмонный резонанс и указаны возможности применения этого эффекта для поиска в окружающей среде следовых количеств молекул, вызывающих сенсибилизацию галоидного серебра к действию света.

Целью настоящей работы является анализ условий эксперимента в [18], оценка чувствительности метода к концентрации молекул красителя в воздухе и поиск возможностей для увеличения чувствительности метода.

\section{ОПИСАНИЕ ОБСУЖДАЕМОГО ЭКСПЕРИМЕНТА}

Суть эксперимента [18] состояла в следующем. Сенсор помещается в замкнутый объем на расстоянии в несколько мм от открытого контейнера, содержащего порошок красителя Арсеназо 3 [18]. Сразу включается освещение - He-Ne лазер с длиной волны 543 нм, длина волны которого попадает в полосу поглощения красителя поверхности детектора. Он представлял трехслойную тонкопленочную структуру серебро-оксид алюминия - йодистое серебро $\left(\mathrm{Ag}-\mathrm{Al}_{2} \mathrm{O}_{3}-\mathrm{AgI}\right)$. Внешняя пленка (AgI) имеет толщину 15 нм. В ходе эксперимента диэлектрическая постоянная внешней пленки регистрируется методом нарушенного полного внутреннего отражения по схеме Кречмана. В камере, заполненной воздухом при обычном атмосферном давлении и комнатной температуре проходила сублимация кристаллов красителя Арсеназо 3. Следовые количества этих молекул адсорбировались на поверхности нанокристалов AgI. Под действием актиничного излучения на поверхности нанокристаллов AgI образовывались кластеры Ag диаметром порядка 50 нм. Поэтому измененялись параметры поверхностного плазмонного резонанса. Измеряемой величиной являлся угол резонансного поглощения [18]. Сразу начинался рост этого угла. Вначале рост был близок к линейному. Скорость роста была больше для больших температур. При прекращении засветки рост резонансного угла прекращался и возобновлялся после возобновления засветки. При отсутствии в камере Арсеназо 3 образование кластеров не регистрировалось при любой экспозиции. Наличие кластеров подтверждено изображением поверхности на атомно-силовом микроскопе, где кластеры видны в виде небольших образований типа неправильного сферического сегмента диаметром около 50 нм, занимающего около $15 \%$ площади нанокристалла. Они видны на всех нанокристаллах, находящихся в поле зрения, имеют близкие размеры, все локализованы неподалеку от края кристалла. Кластеры существуют даже на кристаллах, частично накрытых лежащим сверху кристаллом, и имеют вид открытого кластера, частично накрытого кристаллом из верхнего слоя.

\section{ОБСУЖДЕНИЕ РАССМАТРИВАЕМОГО ЭКСПЕРИМЕНТА}

В литературе [19] рассматривается механизм передачи возбуждения от молекул сенсибилизатора к нанокристаллу галоидного серебра лишь при адсорбированной на поверхности нанокристалла молекуле. Мы также примем это. Тогда наблюдаемая в эксперименте картина может быть интерпретирована следующим образом. Молекулы красителя медленно диффундируют от поверхности порошка. При попадании на поверхность они с некоторой вероятностью $\beta$ адсорбируются поверхностью, возбуждаются освещением и передают возбуждение нанокристаллу. При одном акте передачи восстанавливается один атом серебра [19]. При достаточно быстром восстановлении еще одного атома в том же нанокристалле (не более $24 \mathrm{c}$ ) образуется долго живущий в рамках эксперимента ассоциат двух атомов. После присоединения к ним 
еще атомов (1-4) образуется бесконечно долго живущий кластер, называемый «центр скрытого изображения». Ограничимся при нашей постановке задачи рассмотрением адсорбции одной молекулы, что обосновано ниже. Простой расчет показывает, что при потоке $1 \mathrm{MBT} / \mathrm{cm}^{2}$ плотность числа фотонов, падающих на поверхность при длине волы 543,5 нм

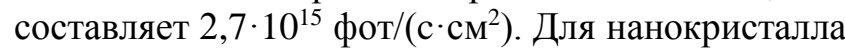
квадратного сечения размером 100 нм это дает $2,7 \cdot 10^{5}$ фотонов падающих на один кристалл за $1 \mathrm{c}$. Поэтому наблюдаемый линейный рост угла резонансного отражения определяется скорее всего скоростью адсорбции молекул красителя на открытой поверхности нанокристаллов иодида серебра.

\section{ОБ ОЦЕНКЕ СКОРОСТИ ОСАЖДЕНИЯ МОЛЕКУЛ КРАСИТЕЛЯ НА ОСНОВЕ \\ МОЛЕКУЛЯРНО-КИНЕТИЧЕСКОЙ ТЕОРИИ}

Опишем процессы, происходящие в эксперименте на основе молекулярно-кинетической теории. В этом случае достаточно хорошо процессы описываются уравнением переноса, в частности, широко применяемым его приближением - уравнением диффузии [20], следствиями из которого мы и воспользуемся [21]. При отсутствии утечек концентрация молекул $n_{a}$ постоянна во всем рассматриваемом объеме и распределение молекул по скоростям соответствует максвелловскому при температуре эксперимента и равномерное по направлениям вектора скорости. Молекулы красителя здесь являются малой тяжелой примесью [21]. При отсутствии адсорбирующих элементов они также равномерно заполняют весь объем при концентрации насыщенных паров красителя для данной температуры. При адсорбции распределение концентраций исказится. В настоящей работе наша цель ориентировочная оценка процессов, поэтому мы примем простейшую схему эксперимента в виде его одномерной модели.

Имеем две параллельные плоскости. Перпендикулярно плоскостям расположена ось $x$. При $x=0$ поддерживается однородная по плоскости плотность молекул красителя $n_{0}$ так, что распределение молекул по скоростям соответствует максвелловскому распределению при температуре эксперимента и равномерное по направлениям. Эту плоскость назовем источником. При $x=L$ находится плоскость детектора с чувствительной поверхностью, обращенной в сторону источника. Эта плоскость поглощает ударяющиеся о нее молекулы красителя с вероятностью $\beta$. Тогда возникнет ток молекул красителя в направлении от источника к детектору. Наша задача - оценить плотность моле- кул красителя $n_{l}$ в плоскости детектора $x=L$ и какова концентрация молекул красителя $n_{0}$ нужна в $x=0$, чтобы за время $t$ получить в плоскости детектора определенную долю $\alpha$ нанокристаллов, на которых образовались кластеры серебра. В стационарных условиях плотность молекул красителя $n(x)$ в точке $x$ не изменяется во времени и ток примесных молекул $j$ будет постоянным [21]

$$
j=-D \frac{d n}{d x}=\text { const }
$$

где $D$ - коэффициент диффузии Арсеназо 3 . Ток частиц $j(x)$, пересекающих слева плоскость с координатой х можно в первом приближении записать как

$$
j(x)=(1 / 2) n(x)\left\langle v_{x}\right\rangle=(1 / 2) n(x)(1 / 2) \bar{v}=(1 / 4) n(x) \bar{v},
$$

где первый множитель $1 / 2$ связан с тем, что около половины молекул распространяется в направлении увеличения $x,\left\langle\mathrm{v}_{\mathrm{x}}\right\rangle$ означает усреднение по направлениям скорости, $\bar{v}-$ средняя величина скорости.

Вследствие постоянства тока из (1) нетрудно получить, что:

$$
n(x)=n_{0}+k x
$$

откуда следует:

$$
\frac{d n}{d x}=\frac{n_{1}-n_{0}}{L} \quad, \quad j=D \frac{n_{0}-n_{1}}{L}
$$

где $n_{1}$ - плотность молекул Арсеназо 3, ударяющихся в плоскость детектора. Выражая для плоскости $x=L$ из равенства (2) $n_{l}=n(L)$ через $j(x)$ и $\bar{v}$, вставляя во второе из выражений (4), решая полученное уравнение относительно $j_{l}=j(L)$ и учитывая (см., например, [21])

$$
D=(1 / 3) \lambda_{h} \bar{v},
$$

где $\lambda_{h}$ - длина пробега с существенным изменением направления движения тяжелой частицы примеси малой концентрации в плотной атмосфере основных легких частиц, получаем:

$$
\begin{gathered}
j_{1}=\frac{1 / 4 n_{0} \bar{v}}{1+(L \bar{v} / D)}=\frac{1 / 4 n_{0} \bar{v}}{1+(3 L) /\left(4 \lambda_{h}\right)} \\
\text { В случае } L>>\lambda_{h}: \\
j_{1}=(1 / 3) \lambda_{h} \bar{v} n_{0} / L
\end{gathered}
$$

\section{ОЦЕНКА ДИФФУЗИОННЫХ ПАРАМЕТРОВ АРСЕНАЗО В ВОЗДУХЕ}

На основе рассмотрения диффузии тяжелой частицы (Арсеназо 3) в воздухе [21] имеем следующее. Молекулярный вес Арсеназо 3 (химическая фоормула: $\mathrm{C}_{22} \mathrm{H}_{18} \mathrm{As}_{2} \mathrm{~N}_{4} \mathrm{O}_{14} \mathrm{~S}_{2}$ ) составляет $m_{\text {ars }}=$ 776,4. Значения ряда параметров Арсеназо 3 найти не удалось, для них мы сделаем оценку. Нам необходимо для расчета длины свободного пробега и 
коэффициента диффузии знать поперечное сечение Арсеназо. Насыпная плотность (плотность порошка) $0,85 \Gamma / \mathrm{cm}^{3}$. При этом там явно имеется много пустот. Поэтому не сильно загрубляя результат мы полагаем плотность равной 1. Далее считаем форму близкой к сферической. В соответствии с [21] исходя из молекулярного веса молекулы Арсеназо 3, принятой нами плотности и сферической формы геометрическое поперечное сечение Арсеназо 3 оценивается как $\sigma_{a r s}=1,18$ нм$^{2}$. При этом в реальности результаты вряд ли отличаются радикально. Наша цель дать лишь приблизительную оценку. Надо оценить длину пробега до существенного изменения направления движения молекулы Арсеназо $3 \lambda_{h}$, ее коэффициент диффузии $D_{a r s}$ и среднюю скорость ее движения $\bar{v}$.

Тяжелая частица находится в окружении быстро движущихся в соответствии с температурой легких частиц воздуха средней массы $\mathrm{m}_{\mathrm{a}}=29$. Поэтому под действием одного соударения импульс молекулы Арсеназо, молекулярный вес которой в 26,8 раза тяжелее среднего молекулярного веса молекул воздуха, существенно изменить нельзя. Для изменения импульса на величину порядка исходного импульса требуется $N_{h}$ ударов [21]:

$$
N_{h}=m_{\text {ars }} / m_{a},
$$

и средняя эффективная длина пробега до существенного изменения направления по порядку величины составляет [21]

$$
\lambda_{h} \approx \sqrt{\frac{m_{\text {ars }}}{m_{a}}} \cdot \frac{1}{\left(n_{0} \sigma_{\text {ars }}\right)},
$$

где $n_{0}$ - концентрация молекул воздуха $\left(2,7 \cdot 10^{19} \mathrm{~cm}^{-3}\right)$, $\sigma_{a r s}-$ сечение взаимодействия молекул арсеназо и воздуха (круг диаметром, равным сумме диаметров обеих молекул, эффективный размер молекулы азота 0,35 нм). Сечение взаимодействия оценено как 2,03 нм². Эффективная длина пробега молекулы арсеназо $\lambda_{h}=94,7$ нм. Т.о. в условиях эксперимента неравенство перед (7) хорошо выполняется от субмикронных расстояний и выше.

Скорость молекул арсеназо определяется выражением:

$$
\bar{v}=\sqrt{8 k T /(\pi m)}=9017 \mathrm{~cm} / \mathrm{c}
$$

и коэффициент диффузии

$$
\begin{gathered}
D_{\text {ars }}=(1 / 3) \lambda_{h} \bar{v}=2.85 \boldsymbol{m s}^{2} / c \\
\text { ОЦЕНКА ПОРОГОВОЙ КОНЦЕНТРАЦИИ } \\
\text { МОЛЕКУЛ АРСЕНАЗО }
\end{gathered}
$$

При малой концентрации молекул красителя возле поверхности за небольшой отрезок времени $d t$ будет адсорбировано небольшое количе- ство молекул $d N<<N$, где $N-$ количество нанокристаллов. Плотность числа нанокристаллов в одном слое на $1 \mathrm{~cm}^{-2}$ составляет $10^{10}$ кристаллов $/ \mathrm{cm}^{2}$. Поскольку среднее число слоев 3 и нанокристаллы не полностью закрывают нижележащие, то можно считать, что $N=3 \cdot 10^{10}$ кристаллов $/ \mathrm{cm}^{2}$. Т.о. вероятность попадания молекул на кристалл составляет $d N / N \ll 1$. После адсорбции при освещении в этих кристаллах начинаются быстрые процессы восстановления металлического серебра. В следующий момент на интервале $d t$ в среднем будет адсорбировано еще $d N$ молекул. Вероятность повторной адсорбции на тот же нанокристалл составляет $(d N / N)^{2}$ и будет крайне мала, Число же нанокристаллов с одной адсорбированной молекулой будет $\sim 2 d N / N$. Поэтому пока не будет покрыта достаточно большая доля нанокристаллов, процесс будет пропорционален времени. Затем, по мере увеличения доли покрытых нанокристаллов линейность процесса должна нарушиться и, в конце концов, должно проявляться насыщение.

С помощью (7) подсчитаем в линейном режиме долю $\alpha$ числа ударившихся о поверхность сенсора молекул красителя на площади $s$ и адсорбированных на нанокристаллах с вероятностью $\beta$ за время $t$

$$
\alpha=j_{1} s t \beta=(1 / 3) \lambda_{h} \bar{v}\left(n_{0} / L\right) s t \beta
$$

Напомним, что $L-$ координата плоскости детектирования, $\mathrm{n}_{0}$ - концентрация молекул Арсеназо 3 в координате $x=0$. Соответствующее выражение для определения значения плотности молекул красителя в плоскости источника

$$
n_{0}=\alpha L /\left((1 / 3) \lambda_{h} \bar{v} s t \beta\right)=\alpha L /(D s t \beta)
$$

Положим, что $s$ средняя площадь нанокристалла и что за время 1 с доля почерневших нанокристаллов $\alpha=0,1$, вероятность адсорбции $\beta=0,1$ и расстояние межу источником и $\mathrm{L}=1$ мм, мы получаем концентрацию частиц во входной плоскости $3,5 \cdot 10^{9} \mathrm{~cm}^{-3}$ или относительная доля молекул примеси $-1,3 \cdot 10^{-10}$. При экспозиции 100 с эти величины уменьшатся на 2 порядка, а при уменьшении расстояния в 10 раз, до 100 мкм, уменьшится еще на порядок и станет $3,5 \cdot 10^{6} \mathrm{~cm}^{-3}$ или относительная доля молекул примеси $-1,3 \cdot 10^{-13}$. В эксперименте расстояния были значительно больше несколько мм. Это в модельной схеме должно увеличить пороговую концентрацию во входной плоскости в несколько раз, но она остается все еще достаточно высокой. Поскольку в реальности плоскости не параллельны, то это должно было приводить к еще большему загрублению чувствительности. 
На основании этих представлений можно оценить плотность частиц в плоскости регистрации. Сопоставляя выражения (2) и (7) получаем

$$
n_{1}=(4 / 3) n_{0}\left(\lambda_{h} / L\right)
$$

Результат вычислений по (14) дает для рассмотренных случаев, что концентрация молекул красителя около плоскости регистрации должна составлять $4,4 \cdot 10^{5} \mathrm{~cm}^{-3}$ или относительная доля молекул примеси в воздухе $-1,65 \cdot 10^{-14}$ (т.е. 0,0165 ppt). При экспозиции 100 с эти величины уменьшатся на 2 порядка и достигнут значений для концентрации $4,4 \cdot 10^{3} \mathrm{~cm}^{-3}$ и для относительной доли молекул примеси $-1,65 \cdot 10^{-16}$. Следует отметить, что по оценкам в кластерах восстановленного серебра содержится около 7\% атомов, содержащихся в нанокристалле. Поэтому химическое проявление поверхности детектора может повысить почернение еще в 10-15 раз, что позволяет еще на порядок повысить чувствительность сенсора.

\section{ЛИТЕР А Т УРА}

1. Обоняние, Большая Советская энциклопедия : в 30 т. Т. 18. Никко - Оболонь. гл. ред. А.М,Прохоров. 3-е изд. М. : Большая Сов. энцикл. 1974.

2. Федоров Ю.А. Индикация наркотических веществ. Специальная техника. 2001. № 5. С. 44-48.

3. Чернышов М.В. Химико-аналитические методы и средства обнаружения взрывчатых веществ для авиационного транспорта. Вопросы оборонной техники. Серия 16: Технические средства противодействия терроризму. 2011. № 7-8. C. 17-30.

4. Стойкова Е.Е., Порфирьева А.В., Евтюгин Г.А. Анализ следовых количеств веществ. Казань, Казанский (Приволжский) федеральный университет им.В.И. УльяноваЛенина. 2010. 72 с.

5. Зоров Н.Б., Попов А.М., Зайцев С.М., Лабутин Т.А. Качественный и количественный анализ объектов окружающей среды методом лазерно-искровой эмиссионной спектрометрии. Успехи химии. 2015. Т. 84. №. 10. С. 1021-1050. DOI: 10.1070/RCR4538?locatt=label:RUSSIAN.

6. Raza N., Hashemi B., Kim K.-H., Lee S.-H., Deep A. Aromatic hydrocarbons in air, water, and soil: Sampling and pretreatment techniques. TrAC Trends in Analytical Chemist. 2018. V. 103. P. 56-73. DOI: 10.1016/j.trac.2018.03.012.

7. Brahim S. Colbern S., Gump R., Grigorian L. Tailoring gas sensing properties of carbon nanotubes. Journal of Applied Physics. 2008. V. 104. P. 024502. DOI: 10.1063/1.2956395.

8. Roberts J.M., Veres P., Warneke C., Neuman J.A., Washenfelder A., Brown S.S., Baasandorj M., Burkholder J.B., Burling I.R., Johnson T.J., Yokelson R.J., de Gouw J. Measurement of $\mathrm{HONO}, \mathrm{HNCO}$, and other inorganic acids by negative-ion proton-transfer chemical-ionization mass spectrometry (NI-PT-CIMS): application to biomass burning emissions. Atmos. Meas. Tech. 2010. V. 3. P. 981-990.

\section{ЗАКЛЮЧЕНИЕ}

Рассмотренный здесь эксперимент [18] показал реальные возможности поверхностного плазмонного резонанса для селективной регистрации сверхмалых концентраций в воздухе при обычных условиях одного из типов красителей и уникальную чувствительность к малым концентрациям, достигающую и возможно превосходящую чувствительность рекордных природных чувствительностей живых организмов. Надо отметить, что с точки зрения применения, схема эксперимента в этой работе [18] хотя и использована в условиях регистрации сверхмалых концентраций вещества вблизи поверхности детектора, но очень неэффективна вследствие доставки пробы диффузией. На основе представленного здесь анализа, можно полагать, что это можно решить простым принудительным перемешиванием или обдувом поверхности сенсора. Это позволит реализовать приведенные выше оценки предельной чувствительности.

\section{REFERENCES}

1. Obonyanie, Bol'shaya Sovetskaya enciklopediya : v 30 t. T. 18. Nikko - Obolon'/ gl. red. A.M,Prohorov. 3-e izd. M. : Bol'shaya Sov. encikl. 1974. (in Russian).

2. Fedorov Yu.A. Indikaciya narkoticheskih veshchestv. Special'naya tekhnika. 2001. N 5. P. 44-48. (in Russian).

3. Chernyshov M.V. Himiko-analiticheskie metody i sredstva obnaruzheniya vzryvchatyh veshchestv dlya aviacionnogo transporta. Voprosy oboronnoj tekhniki. Seriya 16: Tekhnicheskie sredstva protivodejstviya terrorizmu. 2011. N 7-8. P. 17-30. (in Russian).

4. Stojkova E.E., Porfir'eva A.V., Evtyugin G.A. Analiz sledovyh kolichestv veshchestv. Kazan', Kazanskij (Privolzhskij) federal'nyj universitet im.V.I. Ul'yanova-Lenina, 2010. 72 p. (in Russian).

5. Zorov N.B., Popov A.M., Zaytsev S.M., Labutin T.A. Qualitative and quantitative analysis of environmental samples by laser-induced breakdown spectrometry. Russ. Chem. rev. 2015. V. 84 (10). P. 1021-1050. DOI: 10.1070/RCR4538.

6. Raza N., Hashemi B., Kim K.-H., Lee S.-H., Deep A. Aromatic hydrocarbons in air, water, and soil: Sampling and pretreatment techniques. TrAC Trends in Analytical Chemist. 2018. V. 103. P. 56-73. DOI: 10.1016/j.trac.2018.03.012.

7. Brahim S. Colbern S., Gump R., Grigorian L. Tailoring gas sensing properties of carbon nanotubes. Journal of Applied Physics. 2008. V. 104. P. 024502. DOI: 10.1063/1.2956395.

8. Roberts J.M., Veres P., Warneke C., Neuman J.A., Washenfelder A., Brown S.S., Baasandorj M., Burkholder J.B., Burling I.R., Johnson T.J., Yokelson R.J., de Gouw J. Measurement of HONO, HNCO, and other inorganic acids by negative-ion proton-transfer chemical-ionization mass spectrometry (NI-PT-CIMS): application to biomass burning emissions. Atmos. Meas. Tech. 2010. V. 3. P. 981-990. 
9. Bishop C., Halfaya Y., Soltani A., Sundaram S., Li X., Streque J., Ougazzaden A. Experimental Study and Device Design of $\mathrm{NO}, \mathrm{NO}_{2}$, and $\mathrm{NH}_{3}$ Gas Detection for a Wide Dynamic and Large Temperature Range Using Pt/AlGaN/GaN HEMT. IEEE Sensors Journal. 2016. V. 16(18). P. 6828-6838. DOI: 10.1109/jsen.2016.2593050 12_5_bishop2016_IEEE_Sens_ J_N_gas.

10. Chen G., Paronyan T.M., Harutyunyan A.R. Sub-ppt gas detection with pristine graphene. Applied Physics Letters. 2012. V. 101(5). P. 053119. DOI: 10.1063/1.4742327.

11. Kim J., Choi S.-W., Lee J.-H., Chung Y., Byun Y.T. Gas sensing properties of defect-induced single-walled carbon nanotubes. Sensors and Actuators B: Chemical. 2016. V. 228. P. 688-692. DOI: 10.1016/j.snb.2016.01.094.

12. Van Quang V., Van Dung N., Sy Trong N., Duc Hoa N., Van Duy $N$., Van Hieu $N$. Outstanding gas-sensing performance of graphene/ $\mathrm{SnO}_{2}$ nanowire Schottky junctions. Applied Physics Letters. 2014. V. 105(1). P. 013107. DOI: 10.1063/1.4887486.

13. Minh Nguyet Q.T., Van Duy N., Phuong N.T., Trung N.N., Hung C.M., Hoa N.D., Van Hieu N. Superior enhancement of $\mathrm{NO}_{2}$ gas response using $\mathrm{n}-\mathrm{p}$ - $\mathrm{n}$ transition of carbon nanotubes $/ \mathrm{SnO}_{2}$ nanowires heterojunctions. Sensors and Actuators B: Chemical. 2017. V. 238. P. 1120-1127. DOI: 10.1016/j.snb.2016.07.143.

14. Minh Nguyet Q.T, Van Duy N., Hung C.M., Hoa N.D., Van Hieu $N$. Ultrasensitive $\mathrm{NO}_{2}$ gas sensors using hybrid heterojunctions of multi-walled carbon nanotubes and on-chip grown $\mathrm{SnO}_{2}$ nanowires. Appl. Phys. Lett. 2018. V. 112. P. 153110. DOI: 10.1063/1.5023851.

15. Mosier-Boss P.A. Review of SERS Substrates for Chemical Sensing. Nanomaterials. 2017. V. 7(6). P. 142. DOI: 10.3390/nano7060142.

16. Herrera G.M., Padilla A.C., Hernandez-Rivera S.P. Surface Enhanced Raman Scattering (SERS) Studies of Gold and Silver Nanoparticles Prepared by Laser Ablation. Nanomaterials. 2013. V. 3. P. 158-172. DOI: 10.3390/nano3010158.

17. Yang S., Dai X., Stogin B. B., Wong T.-S. Ultrasensitive surfaceenhanced Raman scattering detection in common fluids. Proceedings of the National Academy of Sciences. 2015. V. 113(2). P. 268-273. DOI: 10.1073/pnas.1518980113.

18. Виноградов С.В., Кононов М.А., Савранский В.В., Валянский С.И., Урбайтис М.Ф. Влияние оптической сенсибилизации на поверхностный плазмонный резонанс. Квантовая электроника. 2003. Т. 33. №8. С. 711-713.

19. Шапиро Б.И. Теоретические начала фотографического процесса, М.; Эдиториал УРСС, 2000. 288. С. 35-36.

20. Кейз К., Цвейфель П. Линейная теория переноса. Москва, «Мир», 1972.

21. Попов П.В. Диффузия.: учебно-методическое пособие по курсу Общая физика Москва : МФТИ. 2016. 94 с.
9. Bishop C., Halfaya Y., Soltani A., Sundaram S., Li X., Streque $J$., Ougazzaden A. Experimental Study and Device Design of $\mathrm{NO}, \mathrm{NO}_{2}$, and $\mathrm{NH}_{3}$ Gas Detection for a Wide Dynamic and Large Temperature Range Using Pt/AlGaN/GaN HEMT. IEEE Sensors Journal. 2016. V. 16(18). P. 6828-6838. DOI: 10.1109/jsen.2016.2593050 12_5_bishop2016_IEEE_Sens_ J_N_gas.

10. Chen G., Paronyan T.M., Harutyunyan A.R. Sub-ppt gas detection with pristine graphene. Applied Physics Letters. 2012. V. 101(5). P. 053119. DOI: 10.1063/1.4742327.

11. Kim J., Choi S.-W., Lee J.-H., Chung Y., Byun Y.T. Gas sensing properties of defect-induced single-walled carbon nanotubes. Sensors and Actuators B: Chemical. 2016. V. 228. P. 688-692. DOI: $10.1016 /$ j.snb.2016.01.094.

12. Van Quang V., Van Dung N., Sy Trong N., Duc Hoa N., Van Duy $N$., Van Hieu N. Outstanding gas-sensing performance of graphene/ $\mathrm{SnO}_{2}$ nanowire Schottky junctions. Applied Physics Letters. 2014. V. 105(1). P. 013107. DOI: 10.1063/1.4887486.

13. Minh Nguyet Q.T., Van Duy N., Phuong N.T., Trung N.N., Hung C.M., Hoa N.D., Van Hieu N. Superior enhancement of $\mathrm{NO}_{2}$ gas response using $\mathrm{n}$ - $\mathrm{p}$ - $\mathrm{n}$ transition of carbon nanotubes $/ \mathrm{SnO}_{2}$ nanowires heterojunctions. Sensors and Actuators B: Chemical. 2017. V. 238. P. 1120-1127. DOI: 10.1016/j.snb.2016.07.143.

14. Minh Nguyet Q.T, Van Duy N., Hung C.M., Hoa N.D., Van Hieu $N$. Ultrasensitive $\mathrm{NO}_{2}$ gas sensors using hybrid heterojunctions of multi-walled carbon nanotubes and on-chip grown $\mathrm{SnO}_{2}$ nanowires. Appl. Phys. Lett. 2018. V. 112. P. 153110. DOI: 10.1063/1.5023851.

15. Mosier-Boss P.A. Review of SERS Substrates for Chemical Sensing. Nanomaterials. 2017. V. 7(6). P. 142. DOI: 10.3390/nano7060142.

16. Herrera G.M., Padilla A.C., Hernandez-Rivera S.P. Surface Enhanced Raman Scattering (SERS) Studies of Gold and Silver Nanoparticles Prepared by Laser Ablation. Nanomaterials. 2013. V. 3. P. 158-172. DOI: 10.3390/nano3010158.

17. Yang S., Dai X., Stogin B.B., Wong T.-S. Ultrasensitive surfaceenhanced Raman scattering detection in common fluids. Proceedings of the National Academy of Sciences. 2015. V. 113(2). P. 268-273. DOI: 10.1073/pnas.1518980113.

18. Vinogradov S.V., Kononov M.A., Savranskij V.V., Valyanskij S.I., Urbajtis M.F. Vliyanie opticheskoj sensibilizacii na poverhnostnyj plazmonnyj rezonans. Kvantovaya elektronika. 2003. V. 33. №8. S. 711-713. (in Russian).

19. Shapiro B.I. Teoreticheskie nachala fotograficheskogo processa, M.; Editorial URSS. 2000. 288. S. 35-36. (in Russian)

20. Kejz K., Cvejfel' P. Linejnaya teoriya perenosa. Moskva, «Mir». 1972. (in Russian).

21. Popov P.V. Diffuziya.: uchebno-metodicheskoe posobie po kursu Obshchaya fizika Moskva: MFTI, 2016. 94 s. (in Russian).

Поступила в редакиию (Received) 01.08.2021 Принята к опубликованию (Accepted) 03.09.2021 